\section{Web-Based Interactive Analysis and Animation of Mechanisms}

\author{
Harry H. Cheng \\ Professor \\ Member of ASME \\ e-mail: hhcheng@ucdavis.edu
}

\author{
Dung T. Trang \\ Graduate Research Assistant
}

Integration Engineering Laboratory, Department of Mechanical and Aeronautical Engineering, University of California,

Davis, CA 95616

A Web-based mechanism analysis and animation system is presented in this article. The system is developed in Ch, an embeddable $\mathrm{C} / \mathrm{C}++$ interpreter, and Ch Mechanism Toolkit. It allows users to solve complicated planar mechanism problems conveniently on-line. Users can input the required data to define a mechanism in a Web browser and then click a button for kinematic and dynamic analysis, graphical plotting, and animation for fourbar, crank-slider, geared fivebar, sixbar linkages and cam-follower systems. Examples are provided to illustrate its ease of use as well as its suitability for distance learning. The Web-based system for mechanism analysis and animation is available on the Web at http://www.softintegration/webservices/mechanism/

[DOI: $10.1115 / 1.2161230]$

\section{Introduction}

Due to the rapid advancement of computer technologies, computational methods for analysis and design of multibody mechanical systems are popular in engineering practice. General purpose software packages such as Automated Dynamic Analysis of Mechanical Systems (ADAMS), Dynamic Analysis and Design System (DADS), Working Model [1] were developed to solve complicated engineering design and analysis problems. Although these software programs are powerful, learning curves for these packages are quite steep because users are assumed to have full knowledge of the subject. Special purpose software packages such as the Linkage INteractive Computer Analysis and Graphical Enhanced Synthesis Package (LINCAGES) [2,3], wATT by Heron [4], and Simulation and Analysis of Mechanisms (SAM) by Artas [5] are available for the synthesis and analysis of planar mechanisms. The software package SYNTHETICA [6] can be used for synthesis of spatial mechanisms. Compared with general purpose software packages, these specialized packages are easier to use for mechanism design and analysis. However, these software packages were not intended for Web-based mechanism design and analysis. They are not suitable for use in a network computing environment.

The Web is the most popular medium for passing information because of its versatility across heterogeneous networks and different platforms. It is increasingly popular to use the Web for mechanism design and analysis. For example, cam-follower systems can be designed on-line by entering some design parameters through a Web browser [7]. Control systems can be designed and analyzed on line [8,9]. We have developed a Web-based interactive mechanism design and analysis system $[10,11]$ based on Ch,

Contributed by the Engineering Simulation and Visualization of ASME for publication in the Journal OF COMPUTING AND INFORMATION SCIENCE IN ENGINEERING. Manuscript received July 14, 2004; final manuscript received April 17, 2005. Assoc. Editor: J. Shah. a $\mathrm{C} / \mathrm{C}++$ interpreter [12-14], and Ch Mechanism Toolkit [15]. $\mathrm{Ch}$, conforming to the international $\mathrm{C}$ standard with extensions, contains all salient features of MATLAB for numerical and script computing. The Ch Mechanism Toolkit [15], developed in Ch, is significantly different from other software packages. The users are able to examine the available source code and demo programs provided within the toolkit. The Web-based mechanism design and analysis system, based on this toolkit, is ideal for rapid prototyping. It provides the users with a convenient means to quickly obtain solutions to many mechanism design problems. It is especially suitable for teaching and learning mechanism design and analysis. The Ch Mechanism Toolkit and Web-based system have been used as teaching tools in an undergraduate course "Computer-Aided Mechanism Design" at the University of California, Davis [16]. The Web-based mechanism design and analysis system allows them to verify their solutions quickly with numerical and graphical output as well as animation. The toolkit provides students with the opportunity to study and understand the algorithms and their software implementation for mechanism design and analysis. Additionally, students can use the toolkit's high-level building blocks to develop their own software programs for solving complicated engineering analysis and design problems. For example, students were assigned to develop a complete program for analysis and animation of a Whitworth quick return mechanism [16]. Programs based on Ch and its Mechanism Toolkit for kinematic synthesis of mechanisms are available [17].

In this paper, the Web-based interactive mechanism analysis and animation system will be described. Section 2 presents the major features of the system. Section 3 describes the design of the user interface. Section 4 highlights the software implementation of the system. Section 5 demonstrates its applications with examples. Finally, Sec. 6 provides a summary of Web-based mechanism analysis and animation system. Although the Web-based mechanism analysis and animation system includes many planar mechanisms, only the fourbar linkage is used to illustrate the interface and applications. The ideas presented are applicable to all other mechanisms.

\section{Features of the Web-Based Mechanism Analysis and Animation System}

The Web-based mechanism design and analysis system incorporates many of the features available in the Ch Mechanism Toolkit. With the Web-based system, users can solve complicated mechanism problems using a Web browser without programming. Some tedious and often complicated processes, such as kinematic analysis of a fourbar linkage, can be performed on-line with ease. All the users have to do is to enter parameters in fill-out forms and submit these values for analysis.

Figure 1 shows the initial Web page for mechanism design and analysis on the Web. It contains links to other Web pages for analysis of various planar mechanisms. For example, clicking on the Fourbar linkage link leads to Fig. 2, the primary Web page for fourbar linkage analysis and animation.

Figure 2 shows the main Web page for fourbar linkage analysis, which is separated into three sections: fourbar linkage analysis, synthesis, and special fourbar linkages. The first section consists of links to many Web pages for performing various operations, such as position, velocity, acceleration, and force analysis. Options for plotting the coupler curve and transmission angle is also available for the fourbar linkage. The next section of the Web page is for fourbar linkage synthesis. The synthesis capability of the system is very limited in the current implementation. It can perform three position synthesis of fourbar linkages. Given three sets of angles for the input/output links of a fourbar, this feature can be used to calculate link lengths of the fourbar that satisfies the relation of the input and output links. The last section of Fig. 2 contains links leading to descriptions of special fourbar linkages such as Grashof, non-Grashof, straight line, quick return, and symmetrical linkages as well as singular configurations. There are 


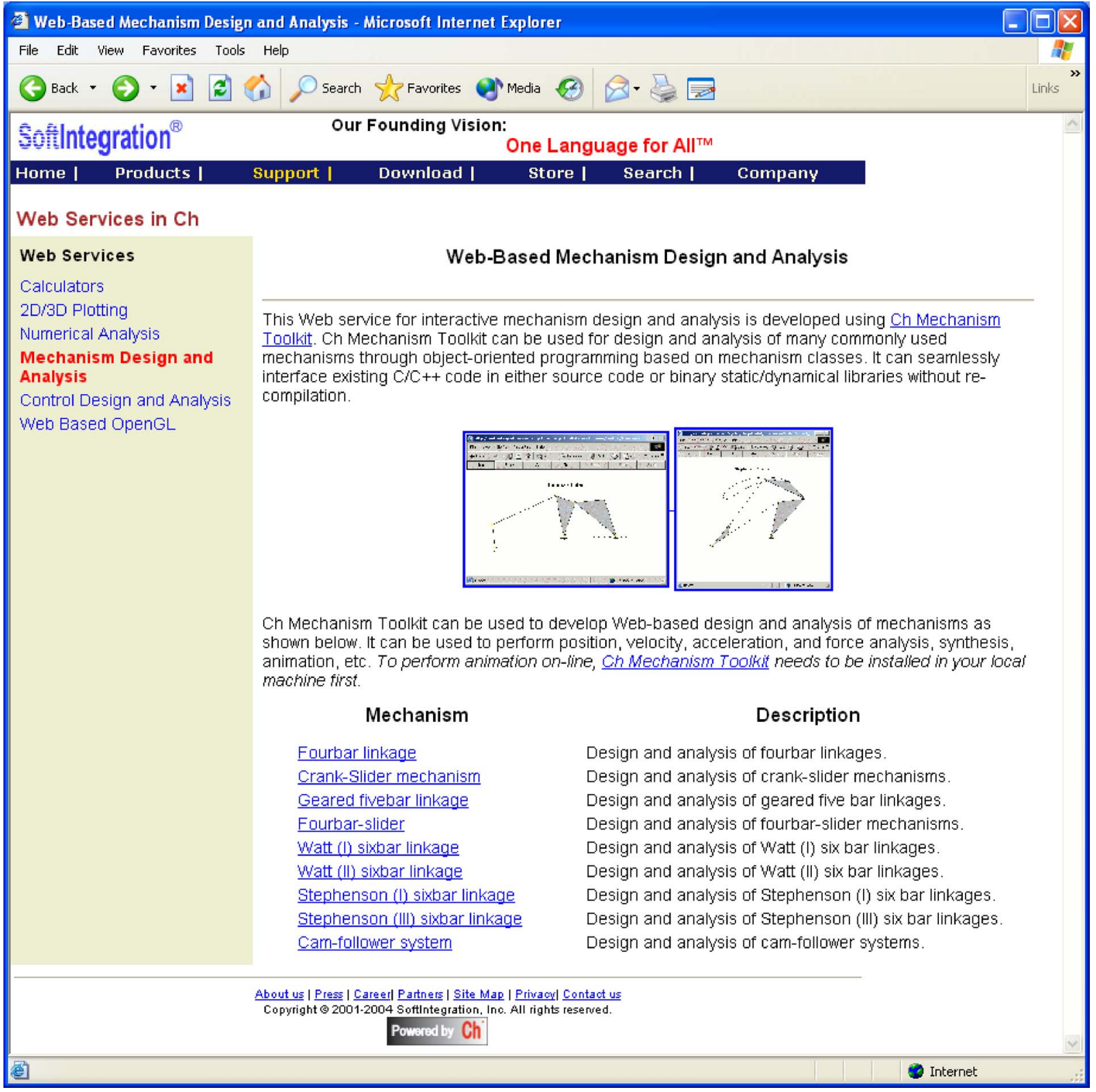

Fig. 1 The main Web page for mechanism design and analysis

a few variety of fourbars for the Grashof, non-Grashof, and straight line linkages. For example, Grashof linkages consists of the crank-rocker, rocker-rocker, rocker-crank, and crank-crank. Some example of straight line linkages are Burmester, Chebychev, Evans, Hoekens, Roberts, and Watt. The Web pages for these special fourbar linkages include a description of each type as well as an animation illustrating their characteristics. For example, the coupler curve for the mid point of the coupler of a Chebychev linkage is a straight line. The linkage has the constraints of $r_{1}: r_{2}: r_{3}=2: 2.5: 1$ and $r_{3}=r_{4}$.

Aside from the fourbar linkage, the Web-based mechanism system also contain tools for analysis of the crank-slider, gearedfivebar, various sixbar linkages, and cam-follower systems as shown in Fig. 1. The available sixbar models include the fourbarslider, STEPHENSON (I) and (III) as well as WATT (I) and (II) sixbar linkages. Operations such as kinematic and dynamic analysis can be performed on the planar linkages mentioned above. For example, given a specific linkage configuration, the angular position, velocity, and acceleration for various linkages can be determined.
Similar to the Ch Mechanism Toolkit, the Web-based system also has some graphical features, such as the ability to simulate the motion of a given mechanism. This animation feature allows the user to visually study the behavior of the mechanism as it rotates through its entire range of motion.

\section{User Interface for Web-Based Mechanism Analysis and Animation}

The interface for the Web-based mechanism design and analysis system consists of a set of Web pages that the user may access to perform various operations. The Web pages were designed to be simple and easy to use. Useful description and implementation information are provided in each page, which are especially useful for learners. For example, Figs. 3 and 4 show the Web page for four bar angular position analysis. Given the angular position of one link, the angular position of the remaining links can be determined. Figure 3 and the top half of Fig. 4 contains a general figure of the fourbar mechanism along with the derivation for calculating 


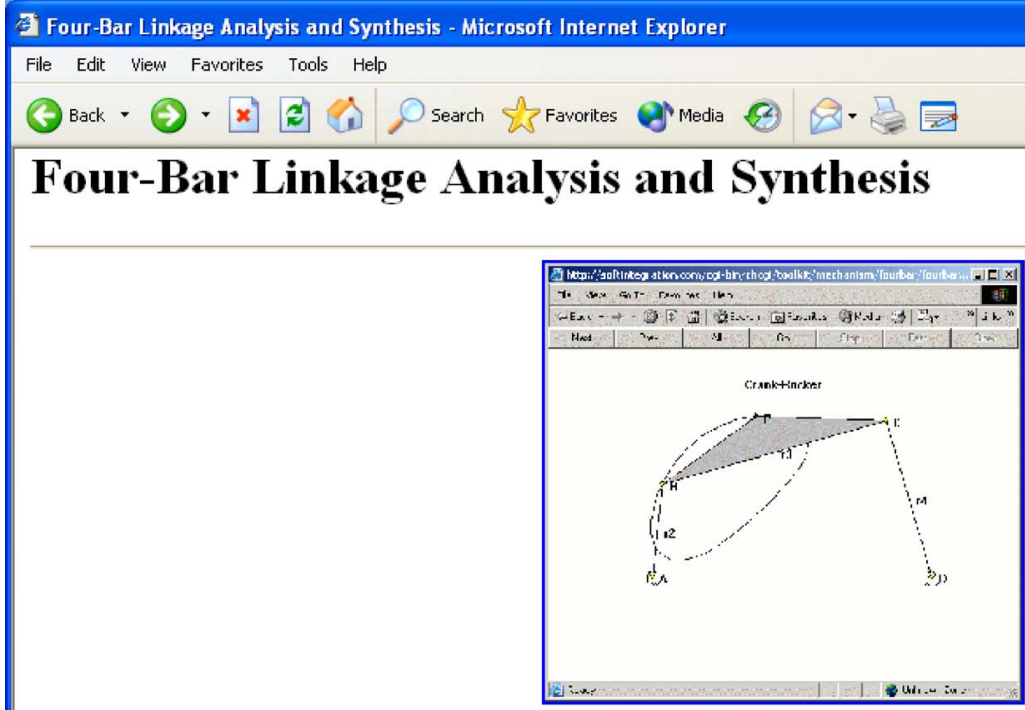

\section{Four-Bar Linkage Analysis}

- Position Analysis

- Coupler Curve Plotting

- Animation

- Transmission Angles

- Transmission Angle Plotting

- Velocity Analysis

- Acceleration Analysis

- Force Analysis

- Kinematic Analysis with Constant Angular Velocity for Link 2

- Dynamic Analysis with Constant Angular Velocity for Link 2

\section{Four-Bar Linkage Synthesis}

- Synthesis with Three Positions

\section{Special Fourbar Linkages with Animation}

- Grashof Linkages

- Non-Grashof Linkages

- Singular Configurations

- Straight Line Linkages

- Quick Return Linkages

- Symmetrical Linkages 
File Edit View Faworites Tools Help

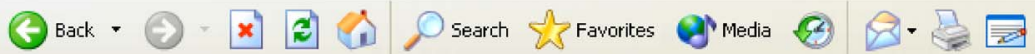

\section{Interactive Four-Bar Linkage Position Analysis}

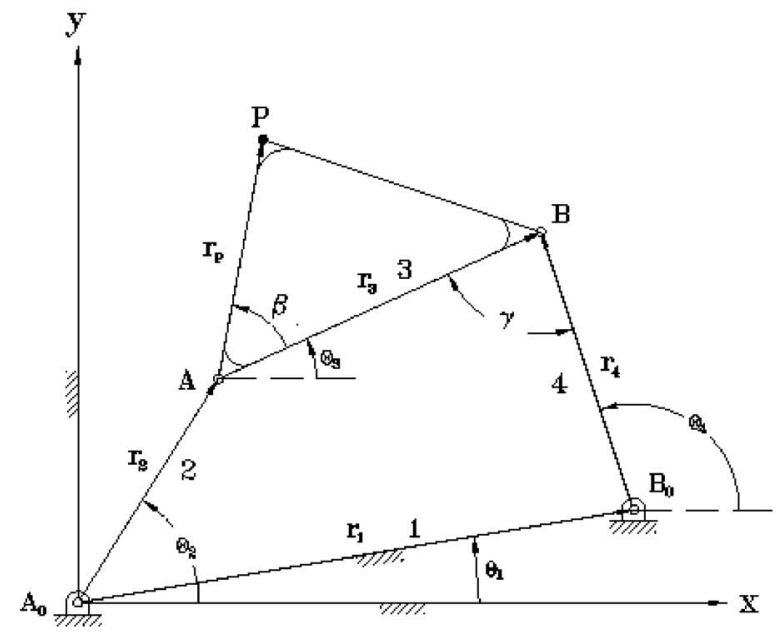

Position analysis begins with formulating the loop-closure equation for the fourbar mechanism shown below

$\mathbf{r} 2+\mathbf{r} 3=\mathbf{r} 1+\mathbf{r} 4$

(1)

Incorporating complex numbers,

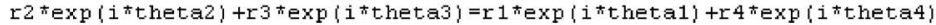

Note link lengths $r 1, \mathrm{r} 2, \mathrm{r} 3$, and $\mathrm{r} 4$ along with theta 1 are constants. Let theta 2 be the independent variable, and theta 3 and theta4 be the dependent variables. Rearanging the equation, we have

$$
r 3^{*} \exp (i * t h e t a 3)-r 4^{*} \exp (i * \operatorname{theta} 4)=r 1^{*} \exp (i * \text { theta } 1)-r 2 * \exp (i * \text { theta }) \text {. }
$$

Let $R 1=r 3$, phi $1=$ theta $3, R 2=-r 4$, phi2 $=$ theta 4 , and $z=(x 3, y 3)=r 1^{*} \exp \left(1^{*}\right.$ theta 1$)-2^{*} \exp \left(1^{*}\right.$ theta2). We now have a general complex equation

$$
R 1 * \exp (i * \operatorname{phi1})+R 2 * \exp (i * \operatorname{phi})=z \text {. }
$$

Angular positions theta 3 and theta 4 can now be solwed for given parameters $r 1, \mathrm{r} 2, \mathrm{r} 3, \mathrm{r} 4$, theta 1 , and theta2. From equation (4) we obtain

$$
\begin{aligned}
& \cos (p h i 1)=(x 3-R 2 \cos (p h i 2)) / R 1 \\
& \sin (p h i 1)=(y 3-R 2 \sin (p h i 2)) / R 1 .
\end{aligned}
$$

Fig. 3 Web-based fourbar position analysis

names and their associated values through the standard input file stream [21]. The CGI programs for Web-based mechanism design and analysis are written in Ch [14], Ch CGI [20], and Ch Mechanism Toolkit [15]. Ch Mechanism Toolkit performs the actual analysis prior to returning the desired output. Before any analysis can be performed, however, a CGI program has to sort out the data sent to it by the Web browser. One such sorting method is to use the getForm( ) function of class CRequest for CGI programming, which allows the program to obtain the value associated with the name indicated by the input argument of string type.

Once all the data have been sorted, an object of the appropriate mechanism class is created to begin the analysis process. The sorted data are then used as parameter values to specify the appropriate linkage mechanism (fourbar, crank-slider, or geared fivebar, etc.). Finally, the defined mechanism is evaluated, and the desired output s shown on the Web browser. Numerical outputs are automatically written into the browser window, whereas, the data for plotting and animation are sent to the standard output stream. The Web browser receives the data and converts to the proper graphical output. Plots are initially generated using the $\mathrm{Ch}$ CPlot class in the CGI programs and sent to the Web browser in Portable Network Graphics (PNG) file format. The animation data are generated in the same manner. However, it requires the QuickAnimation plug-in to display the animation in the Web browser window.

\section{Examples of Web-Based Mechanism Analysis and Animation}

In this section, two examples will used to illustrate some of the features and applications of the Web-based mechanism design and analysis system.

5.1 Example 1. Problem Statement: The link lengths of a 
Substituting these results into the trig identity $\sin ^{\wedge} 2($ phi1 $)+\cos ^{\wedge} 2($ phi1 $)=1$ and simplifying we obtain

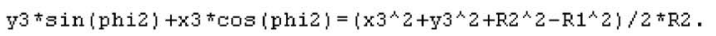

From this equation we can obtain formulas for phi1 and phi2

phi2 $=\operatorname{atan} 2(y 3, x 3) \pm \operatorname{acos}\left(\left(x 3^{\wedge} 2+y^{\wedge} 3^{\wedge} 2+R 2^{\wedge} 2-R 1^{\wedge} 2\right) / 2 * R 2 * \operatorname{sqrt}\left(x 3^{\wedge} 2+y 3^{\wedge} 2\right)\right)$

$$
\begin{gathered}
\text { phi1=atanz }(\sin (p h i 1), \cos (p h i 1)) \\
=a \tan 2\left(\left(\mathrm{y}^{3}-\mathrm{R} 2 \sin (\mathrm{phi} 2)\right) / \mathrm{R} 1,(\mathrm{x} 3-\mathrm{R} 2 \cos (\mathrm{phi})) / \mathrm{R} 1\right) . \quad(9)
\end{gathered}
$$

Similar equations can be derived assuming either theta 3 or theta 4 is known with the other two angles as parameters

Please enter link lengths, thetal and one other known angle to find the other two angles

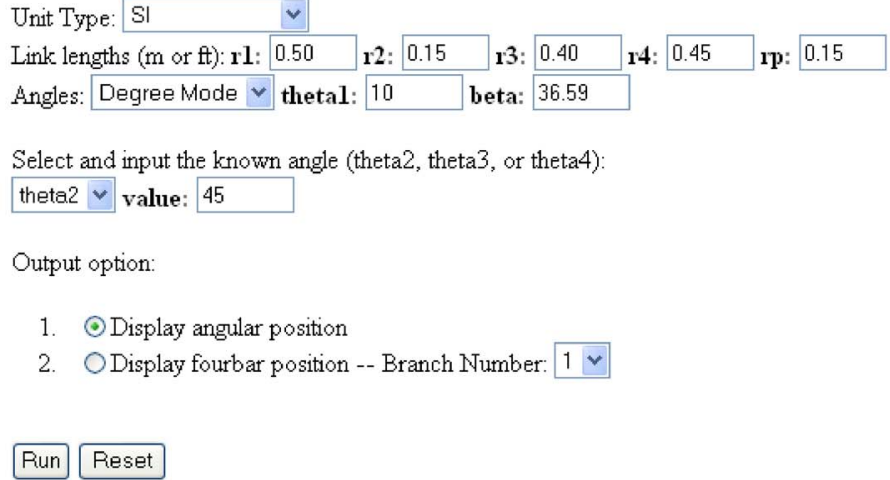

Fig. 4 Web-based fourbar position analysis (contd.)

fourbar linkage shown in Fig. 3 are given as follows: $r_{1}=12 \mathrm{~cm}$, $r_{2}=4 \mathrm{~cm}, r_{3}=12 \mathrm{~cm}$, and $r_{4}=7 \mathrm{~cm}$. The phase angle for the ground link is $\theta_{1}=10 \mathrm{deg}$. The coupler point $P$ is defined by the distance $r_{p}=5 \mathrm{~cm}$ and constant angle $\beta=20 \mathrm{deg}$. Determine the angular positions $\theta_{3}$ and $\theta_{4}$ as well as the position for coupler

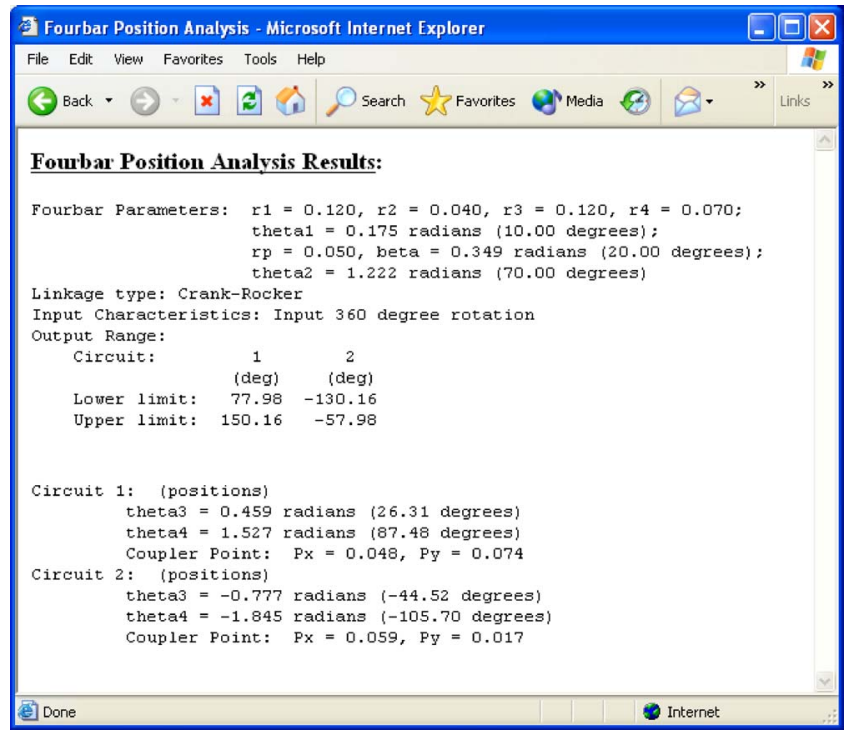

Fig. 5 Numerical output of the Web-based fourbar position analysis

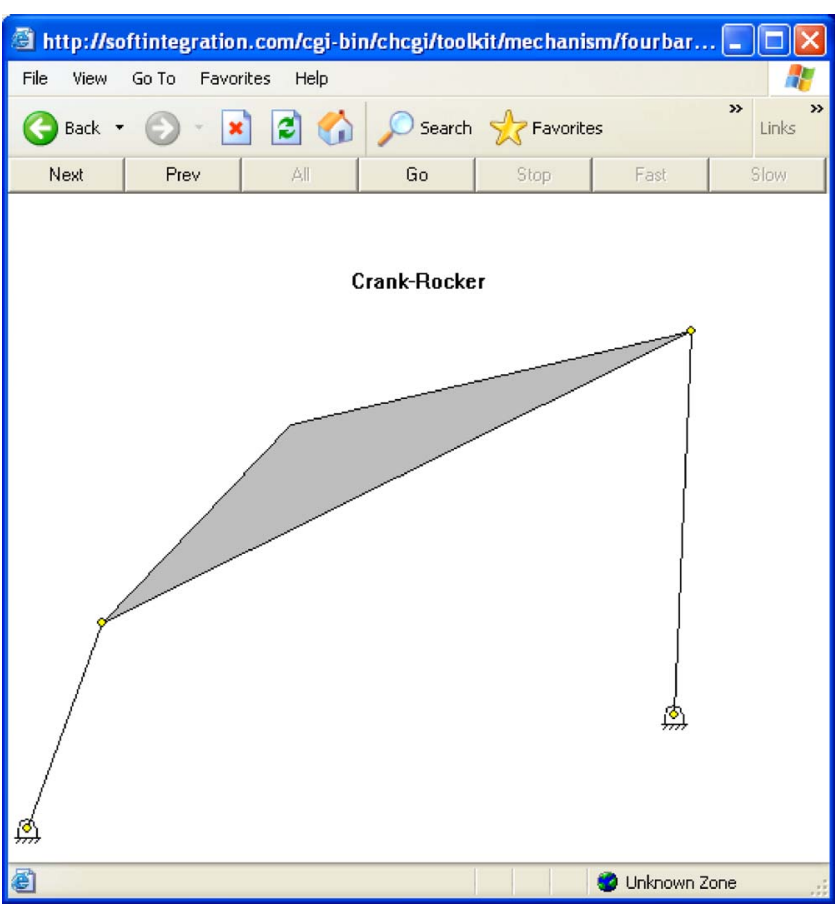

Fig. 6 The graphical output for branch 1 of the Web-based fourbar position analysis 


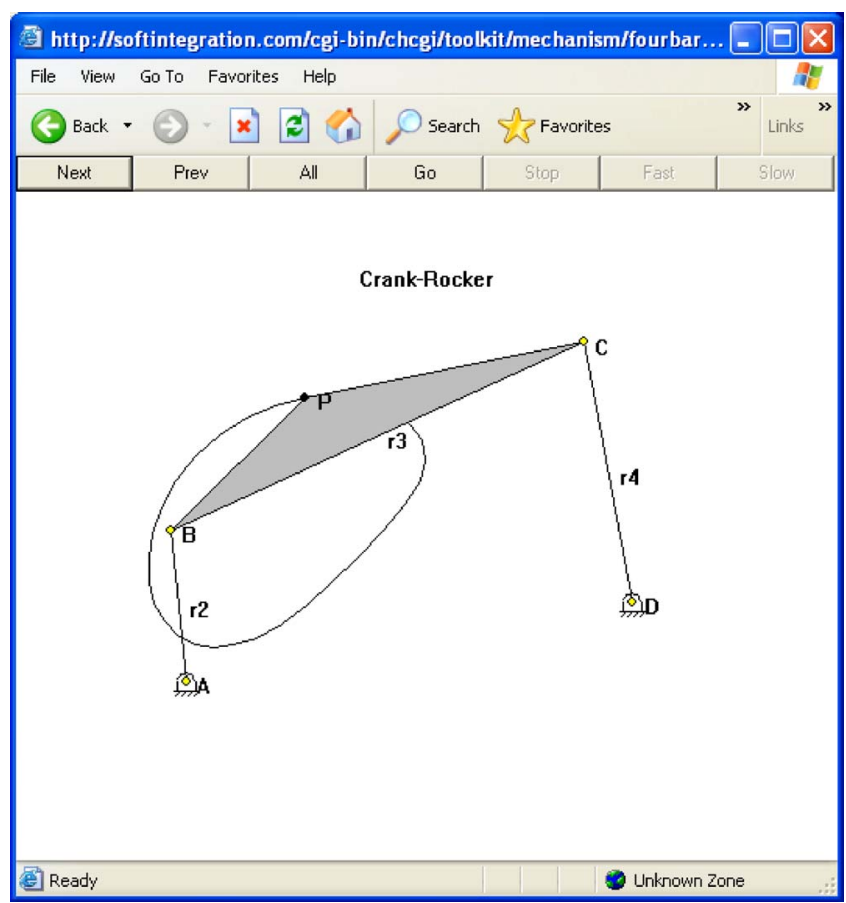

Fig. 7 The single frame of the Web-based fourbar animation

point $P$ when the input angle $\theta_{2}=70 \mathrm{deg}$.

This problem can be quickly solved by the angular position analysis Web page introduced earlier as shown in Fig. 4. After inputting the given parameters into the fill-out form, the analysis can be performed by simply clicking the Run button. The numerical results of the analysis are shown in Fig. 5. In addition to the values for $\theta_{3}$ and $\theta_{4}$, the numerical output also displays the range of motion for the input/output links as well as the coupler point position $\mathrm{P}$ for each circuit of the fourbar. As shown in Fig. 5, the fourbar linkage is a crank-rocker with two circuits. The input

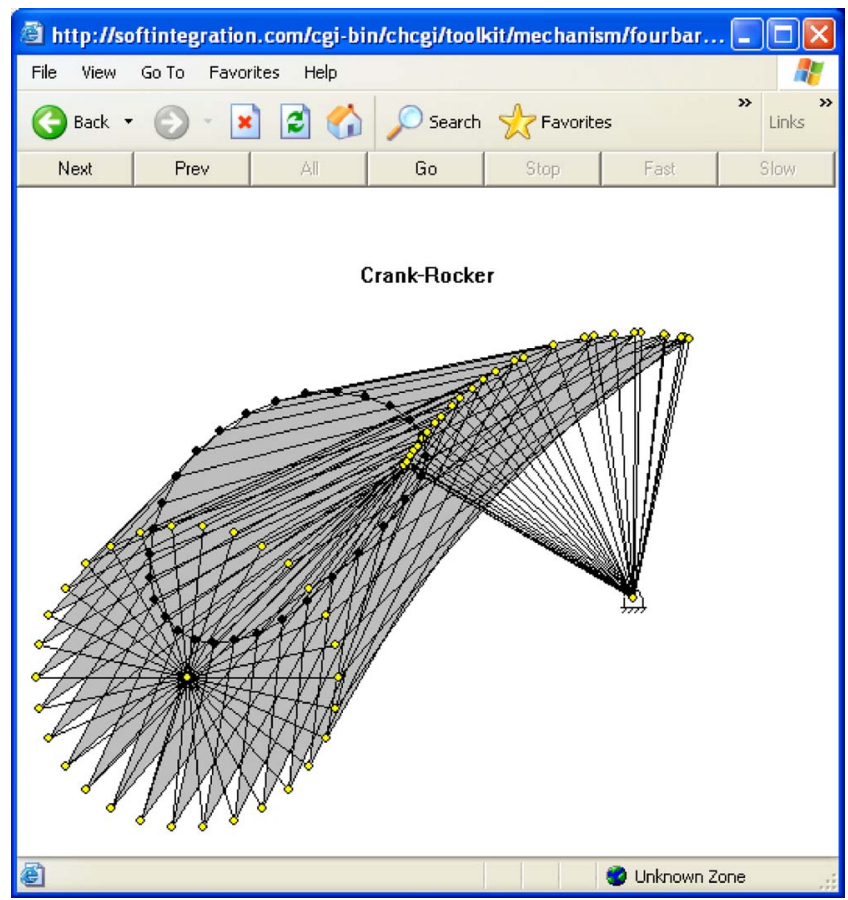

Fig. 8 All frames of the Web-based fourbar animation range for $\theta_{2}$ is a complete rotation with $360 \mathrm{deg}$. The output range for $\theta_{4}$ is $77.98 \mathrm{deg} \leqslant \theta_{4} \leqslant 150.15 \mathrm{deg}$ and $-130.16 \mathrm{deg} \leqslant \theta_{4} \leqslant$ $-57.98 \mathrm{deg}$ for the first and second circuits, respectively. At when $\theta_{2}$ is $70 \mathrm{deg}, \theta_{3}, \theta_{4}$, and the coupler point are $26.31 \mathrm{deg}$, $87.48 \mathrm{deg}$, and $(0.048 \mathrm{~cm}, 0.074 \mathrm{~cm})$ for the first circuit and $-44.52 \mathrm{deg},-105.70 \mathrm{deg}$, and $(0.059 \mathrm{~cm}, 0.017 \mathrm{~cm})$ for the $\mathrm{sec}-$ ond circuit, respectively.

If desired, the user may also choose to graphically display the configuration of the fourbar linkage, such as Fig. 6, which shows the configuration of the specified four par for the first branch. Note that the four bar specified in the above problem statement is a crank-rocker, which means that there are two distinct circuits for each input link position. Thus, the user can display the second circuit by selecting 2 for the branch number prior to running the Web-based application in Fig. 4.

5.2 Example 2. Problem Statement: Simulate the motion of the fourbar linkage defined in Example 1 for its entire range of motion.

As in the first example, the above problem can easily be handled by the Web-based mechanism system. Similar to the fourbar position analysis Web page, the animation Web page also contains buttons, pull-down menus, and text boxes for users to specify the parameters of the fourbar linkage. The last option allows the user to indicate the branch number of the fourbar to animate. For typical fourbar linkages, such as the crank-rocker used in these two examples, there are two possible geometric inversions. However, for a fourbar rocker-rocker mechanism, there are a total of four possible geometric inversions. Figure 7 shows one frame of animation for the first geometric inversion of the fourbar crank-rocker mechanism, whereas Fig. 8 is an overlay of all the frames of the animation.

\section{Conclusion}

The Web-based mechanism analysis and animation system, based on the Ch Mechanism Toolkit, has been presented in this paper. This system consists of multiple user-friendly Web pages for the interface and CGI programs to perform the relevant analysis. The Web-based system is simple and easy to use. If the user ever enters an invalid value, an error message will be displayed to indicate the problem. The Ch Mechanism Toolkit and its Webbased extension can be used to solve various mechanism analysis problems. It can compute the angular positions, velocities, and accelerations of the individual links of mechanisms such as the fourbar, crank-slider, geared-fivebar, sixbar linkages, and camfollower systems. Furthermore, it can also provide graphical output, such as animation, through the Web. The Ch Mechanism Toolkit and its Web-based system are not only useful for solving practical engineering problems, but also ideal for distance learning. The ideas and concepts presented in the Ch Mechanism Toolkit and Web-based system can be applied to solve many other mechanism designs and analysis problems.

\section{References}

[1] Working Model User's Guide, Knowledge Revolution, 1989.

[2] Erdman, A. G., and Gustafson, J. E., 1981, "LINCAGES: Linkage INteractive Computer Analysis and Graphically Enhanced Synthesis Package," ASME Paper, No. 77-DET-5.

[3] Erdman, A. G., and Riley, D. R., 1981, "Computer-Aided Linkage Design Using the LINCAGES Package," ASME Paper, No. 81-DET-121.

[4] WATT 1.6 User's Guide, Heron Technologies, 2002 (online) available at http:// www.herontechnologies.com

[5] SAM 5.0 User's Guide, Artas\#x2014;Engineering Software, 2003 (online) available at http://www.artas.nl

[6] Perez, A., Su, H. J., and McCarthy, M., 2004, "SYNTHETICA 2.0:Software for the Synthesis of Constrained Serial Chains," in Proceedings of the ASME Design Engineering Technical Conferences, No. DETC2004/57524, Salt Lake City, Utah, September 2004.

[7] Larson, J., and Cheng, H. H., 2000, "Object-Oriented Cam Design Through the Internet," J. Intell. Manuf., 11, pp. 515-534.

[8] Zhu, Y., Chen, B., and Cheng, H. H., 2003, “An Object-Based Software Package for Interactive Control System Design and Analysis," J. Comput. Inf. Sci. 
Eng., 3, pp. 366-371.

[9] Yu, Q., Chen, B., and Cheng, H. H., 2004, "Web-Based Control System Design and Analysis," IEEE Control Syst. Mag., 24, pp. 45-57.

[10] Cheng, H. H., and Trang, D. T., 2004, "Web-Based Mechanism Design and Analysis," in Proceedings of the ASME 28th Mechanism and Robotics Conference, No. DETC2004-57594, Salt Lake City, Utah, Sept. 2004.

[11] Web-Based Mechanism Design and Analysis, Softintegration, Inc. (online) available at http://softintegration.com/webservices/mechanism/

[12] Cheng, H. H., 1993, "Scientific Computing in the Ch programming language," Sci. Prog., 2, pp. 49-75.

[13] Cheng, H. H., 1995, "Extending C and FORTRAN for Design Automation," ASME J. Mech. Des., 117, pp. 390-395.

[14] $\mathrm{Ch}$, an Embeddable $\mathrm{C} / \mathrm{C}++$ Interpreter (online) available at http:// www.softintegration.com

[15] Ch Mechanism ToolKit User's Guide, Softintegration, Inc. (Online) available at http://www.softintegration.com/products/toolkit/mechanism/

[16] Design and Analysis of Whitworth Quick Return Mechanism (online) available at http://iel.ucdavis.edu/projects/mechanism/quickreturn

[17] E. Pennestri, Kinematic Synthesis of Mechanisms (online) available at http:// www.ingegneriameccanica.org/mechanisms.htm

[18] The Common Gateway Interface, MCSA Software Development Group, 1996

[19] Cheng, H. H., 1996, "CGI Programming in C," C/C ++ Users Journal, pp. $17-21$.

[20] The Ch Language Environment CGI ToolKit User's Guide, Softintegration, Inc. (online) available at http://www.softintegration.com/products/toolkit/cgi/

[21] Katwyk, K. V., and Cheng, H. H., 1997, "XLINKAGE: A Web-Based Analysis and Simulation Tool for Planar Mechanical Systems," in Proceedings of the ASME Design Engineering Technical Conferences, No. DETC97/DAC-3863, Sacramento, California. 